\title{
Effect of Internal Architecture on the Assembly of Soft Particles at Fluid Interfaces
}

\author{
Jacopo Vialetto,* Fabrizio Camerin,* Fabio Grillo, Shivaprakash N. Ramakrishna, Lorenzo Rovigatti, \\ Emanuela Zaccarelli,* and Lucio Isa*
}

Cite This: ACS Nano 2021, 15, 13105-13117

Read Online

ABSTRACT: Monolayers of soft colloidal particles confined at fluid interfaces are at the core of a broad range of technological processes, from the stabilization of responsive foams and emulsions to advanced lithographic techniques. However, establishing a fundamental relation between their internal architecture, which is controlled during synthesis, and their structural and mechanical properties upon interfacial confinement remains an elusive task. To address this open issue, which defines the monolayer's properties, we synthesize core-shell microgels, whose soft core can be chemically degraded in a controlled fashion. This strategy allows us to obtain a series of

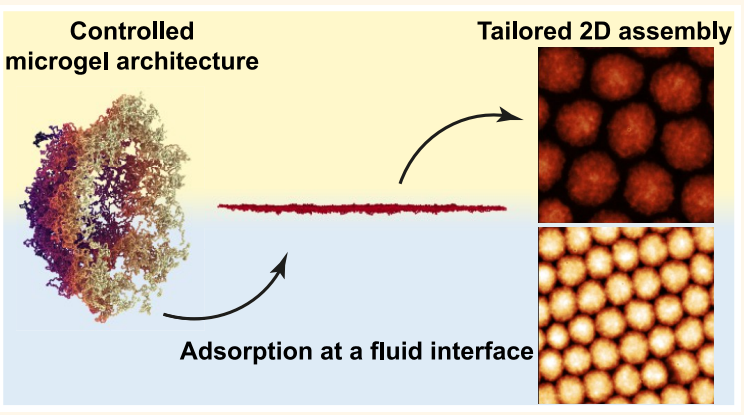
particles ranging from analogues of standard batch-synthesized microgels to completely hollow ones after total core removal. Combined experimental and numerical results show that our hollow particles have a thin and deformable shell, leading to a temperature-responsive collapse of the internal cavity and a complete flattening after adsorption at a fluid interface. Mechanical characterization shows that a critical degree of core removal is required to obtain soft disk-like particles at an oilwater interface, which present a distinct response to compression. At low packing fractions, the mechanical response of the monolayer is dominated by the outer polymer chains forming a corona surrounding the particles within the interfacial plane, regardless of the presence of a core. By contrast, at high compression, the absence of a core enables the particles to deform in the direction orthogonal to the interface and to be continuously compressed without altering the monolayer structure. These findings show how fine, single-particle architectural control during synthesis can be engineered to determine the interfacial behavior of microgels, enabling one to link particle conformation with the resulting material properties.

KEYWORDS: pNIPAM microgels, liquid interface, modeling, self-assembly, colloidal particles

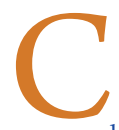
olloidal particles are ideal building blocks to obtain micro- and nanostructured materials whose properties can be tailored by engineering interparticle interactions. ${ }^{1,2}$ Using soft colloids, the coupling between particle architecture and deformability in different environments (e.g., in bulk suspensions or following confinement at interfaces, under different temperature or $\mathrm{pH}$ conditions, etc.) enables a rich behavior and imparts functionalities unattainable with mechanically rigid, i.e., "hard", particles. ${ }^{3,4}$ Microgels, colloidal particles comprising an internally cross-linked network formed by a water-soluble polymer, have emerged as a prominent choice with facile synthesis and versatile applications. ${ }^{5-7}$ Among the latter, materials comprising microgel monolayers confined at solid or fluid interfaces, such as air-water or oilwater interfaces, present both high potential and important challenges. The versatility in their assembly and their responsiveness enable microgel monolayers to be used as substrates for cell culture and harvesting, ${ }^{8}$ or in sensing, ${ }^{9}$ optics, ${ }^{10}$ and patterning ${ }^{11}$ applications. Additionally, self- assembly at fluid interfaces and transfer to solid substrates allow using microgel monolayers as tunable nanolithography masks, ${ }^{12,13}$ whereas the direct use of microgels at the fluid interface can be exploited to obtain smart responsive foams ${ }^{14}$ or emulsions. ${ }^{15}$

In spite of the practical interest, currently, there are no fundamental guidelines that connect synthesis procedures in bulk with the resulting single-particle properties and collective behavior of the microgels upon interfacial adsorption. Moreover, it is still unclear how different three-dimensional (3D) shapes of the microgels in suspension translate into their

Received: March 23, 2021

Accepted: July 23, 2021

Published: July 30, 2021

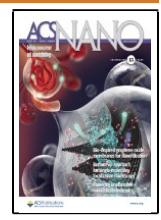


a

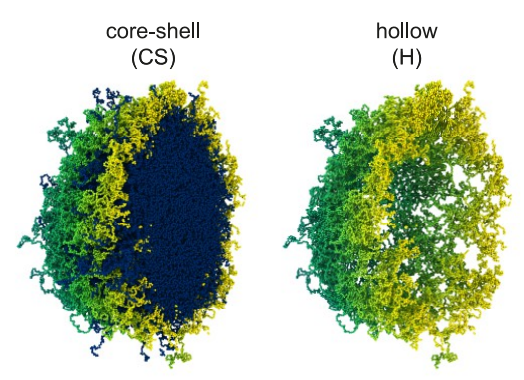

C

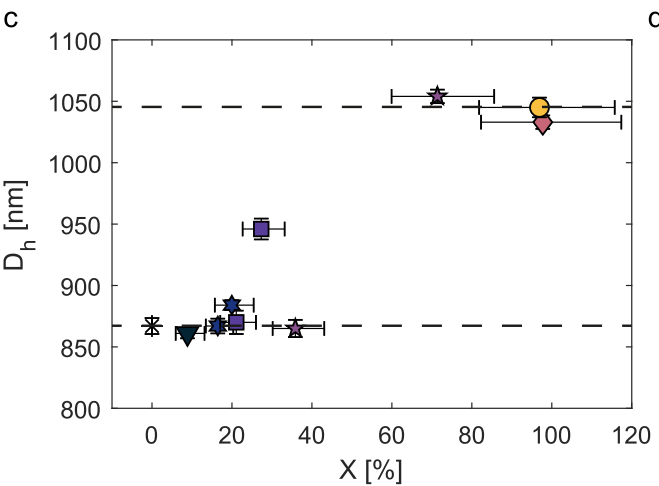

b

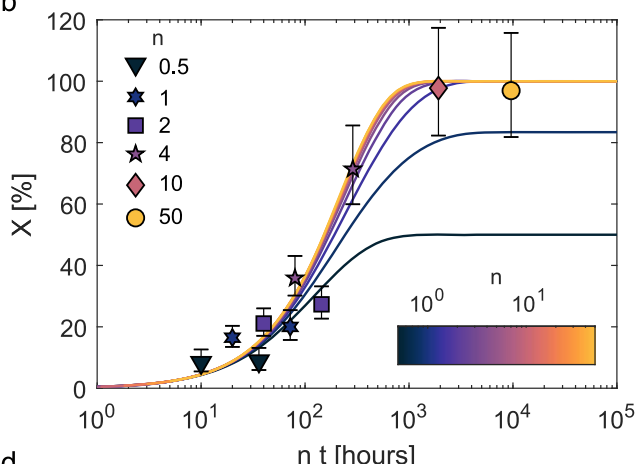

d

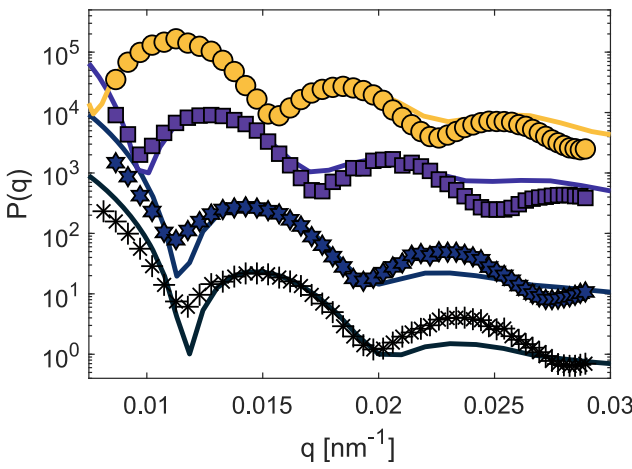

Figure 1. Core-shell and hollow microgels in bulk aqueous suspensions: core degradation process. (a) Simulation snapshots of the cross section of a core-shell microgel before and after the removal of the inner core. The core with the DHEA cross-linker is colored in blue, and the outer shell with the BIS cross-linker is colored in green/yellow. (b) Extent of core removal $X$ as a function of $n^{\bullet} t$, where $n$ is the $\mathrm{NaIO}_{4}$ to DHEA molar ratio and $t$ is the reaction time. The symbols indicate the values of $X$ estimated from the experimental form factors (see Materials, Models, and Methods), whereas the lines show eq 2 fitted to the experimental $X$ for different values of $n$. (c) Hydrodynamic diameter $D_{\mathrm{h}}$ of core-shell microgels as a function of the extent of core removal $X$. The horizontal dashed lines emphasize the sudden transition to a larger dimension upon removal of a critical core fraction. (d) Experimental (symbols) and numerical (lines) form factors as a function of the wavenumber $q$. The experimental form factors are for $n \cdot t=0,20,144$, and 9600 , whereas the numerical ones are calculated for different number densities of the core microgel: $\rho \approx 0.08,0.06$, and $0.01 \sigma^{-3}$ (from the bottom to the top), where $\sigma$, representing the monomer size, is the unit length in the simulations. The yellow circles correspond to the hollow microgel $(X=100 \%)$. The form factors are arbitrarily shifted along the $y$-axis for visual clarity. The symbols in (c) and (d) correspond to the same values of $n$ shown in (b), with * black symbols indicating the core-shell microgel prior to core removal $(n=0)$.

new morphology at the interface and how their bulk mechanical properties are transferred to the interface. ${ }^{16-19}$

The vast majority of microgels are prepared by one-pot precipitation polymerization, which leads to the formation of particles with a radially decreasing cross-linking density profile in bulk aqueous suspensions. ${ }^{20,21}$ As the particles adsorb to a fluid interface, surface tension and the different solubility of the polymer in the two phases cause a reconfiguration of the polymer network. ${ }^{22-24}$ These standard microgels flatten out at the interface, with the more loosely cross-linked polymer chains at the particle periphery creating a thin corona, which surrounds the particle within the interfacial plane. Nowadays, various synthetic procedures allow realizing microgels with different architectures, such as homogeneously crosslinked $^{25,26}$ or hollow ones. ${ }^{23,27-30}$ The case of hollow microgels, that is, particles made of a polymeric shell surrounding an empty core, is particularly appealing. Their peculiar responses to swelling and compression in $3 \mathrm{D},{ }^{31-33}$ specifically relating to the conformation and retention of the inner cavity, poses untapped opportunities to tailor their assembly at fluid interfaces.

In this article, we address the general question of how the internal architecture of a microgel affects its bulk and interfacial properties by selecting and characterizing a model system that allows us to tune the particle inner structure from the one of a standard batch-synthesized microgel to a hollow one. To this end, we synthesize core-shell microgels, where the inner microgel core can be chemically degraded in a controlled fashion. ${ }^{28}$ We experimentally and numerically follow the progressive removal of the core and evidence how it affects both the swelling-deswelling transition in bulk and the particle morphology at the interface. We then focus on the collective response of hollow microgels under compression at the interface. In particular, our final hollow particles present a significantly thinner and softer shell compared to those in existing literature, ${ }^{23,29,33-35}$ which discloses a rich mechanical and structural behavior. Overall, our approach allows precisely linking single-particle properties to their interfacial assembly in order to tailor the two-dimensional (2D) assembly of soft colloids.

\section{RESULTS AND DISCUSSION}

Characterization of the Hollow Microgels in Bulk. Core Degradation. The hollow microgels used in this study are synthesized by means of a two-step polymerization procedure, followed by a degradation process. ${ }^{28} \mathrm{We}$ first obtain a standard microgel by free-radical precipitation polymerization using DHEA as a cross-linker, and afterward, we grow a second polymeric shell on top using methylenebis(acrylamide) (BIS) as a cross-linker, forming a core-shell 
particle with a thin interpenetrated network in the interfacial region (see Materials, Models, and Methods for a detailed description of the synthesis). By exposing the core-shell (CS) microgels to $\mathrm{NaIO}_{4}$, the DHEA cross-linkers in the core (C) can be degraded, leading to the progressive formation of hollow $(\mathrm{H})$ microgels, as represented by the simulation snapshots of Figure 1a.

We study the kinetics of the degradation of the core as a function of the time $t$ and the initial $\mathrm{NaIO}_{4}$ to DHEA molar ratio $n=\frac{\left[\mathrm{NaIO}_{4}\right]_{0}}{[\mathrm{DHEA}]_{0}}$ via ex situ static light scattering (SLS) and dynamic light scattering (DLS) measurements. In particular, we vary $n$ in the range of $0.5-50$ and $t$ in the range of 20-192 $h$ and define the degree of core removal as $X(n, t)=\frac{M_{c}^{0}-M_{c}(n, t)}{M_{c}^{0}}$. Here, $M_{c}^{0}$ and $M_{c}(n, t)$ are the mass of the core before and after the reaction with $\mathrm{NaIO}_{4}$, respectively. These are estimated from the microgels' radial density profiles, which we extract by fitting the form factors for different values of $n$ and $t$, as described in Materials, Models, and Methods and reported in Figures $S 1$ and S2. The symbols in Figure $1 \mathrm{~b}$ represent the experimental $X(n, t)$ for different values of $n$. When plotted against the product $n \cdot t, X$ exhibits a sigmoidal trend, with larger $n \cdot t$ leading to increasingly higher $X$ up to $n \cdot t$ $\simeq 10^{3} \mathrm{~h}$, after which $X$ reaches a plateau at $\simeq 100 \%$.

To rationalize such kinetics, we model the degradation of the core as a bimolecular and stoichiometric reaction between the vicinal diol group in the DHEA polymer network and $\mathrm{NaIO}_{4}{ }^{28}$ The reaction $\mathrm{DHEA}_{(\mathrm{s})}+\mathrm{NaIO}_{4(1)} \rightarrow \mathrm{C}_{(1)}$ leads to a soluble product $\mathrm{C}_{(1)}$ that is washed out during the postreaction cleaning step. By assuming the reaction follows a first-order kinetics with respect to the concentration of both DHEA and $\mathrm{NaIO}_{4}$, perfect mixing within the reaction vessel, and a $1: 1$ linear correlation between the degree of core removal and the cleavage of the vicinal diol in the DHEA molecules, $X$ is prescribed by the following differential equation:

$$
\frac{\mathrm{d} X}{\mathrm{~d} t}=k[\mathrm{DHEA}]_{0}(1-X)(n-X)
$$

where $k$ is the reaction rate constant. This equation admits two analytical solutions depending on whether $n=1$ or $n \neq 1$ :

$$
X(n, t)=\left\{\begin{array}{l}
\frac{n\left(\mathrm{e}^{(n-1)[\mathrm{DHEA}]_{0} k t}-1\right)}{n \mathrm{e}^{(n-1)[\mathrm{DHEA}]_{0} k t}-1}, n \neq 1 \\
\frac{[\mathrm{DHEA}]_{0} k t}{[\mathrm{DHEA}]_{0} k t+1}, n=1
\end{array}\right.
$$

It is important to remark that, within this framework, for a given initial DHEA concentration $[\mathrm{DHEA}]_{0}$, varying $n$ is not equivalent to varying $t$ as $X(n, t)$ is not a function of the product $n \cdot t$. This emphasizes the importance of studying the effects of varying both $n$ and $t$ separately. Nonetheless, for $n \gg$ $1, X(n, t)$ can be approximated to a sole function of the product $n \cdot t$ as $X(n, t) \sim\left(\mathrm{e}^{n k t}-1\right) / \mathrm{e}^{n k t}$.

The fitting of the experimental $X(n, t)$ with eq 2 is plotted in Figure $1 \mathrm{~b}$ against $n \cdot t$, giving an excellent description of the experimental data $\left(R^{2}=0.96\right)$ with a value of $k=1.33 \times 10^{-2}$ $\pm 4 \times 10^{-3} \mathrm{~m}^{3} \mathrm{~mol}^{-1} \mathrm{~h}^{-1}$.

The degree of core removal correlates with the microgels' hydrodynamic diameter $D_{\mathrm{h}}$, as measured by DLS. The standard DHEA-cross-linked (core) microgels prior to the growth of the BIS shell have $D_{\mathrm{h}}=640 \mathrm{~nm}$ at $22{ }^{\circ} \mathrm{C}$ (blue triangle in Figure
S3). After the second polymerization step, the $D_{\mathrm{h}}$ of the coreshell particle reaches $870 \mathrm{~nm}\left(22{ }^{\circ} \mathrm{C}\right)$. We then calculate the thickness of the BIS shell as $\left(D_{\mathrm{h}}(C S)-D_{\mathrm{h}}(C)\right) / 2$, obtaining a value of $115 \mathrm{~nm}$. After reaction with $\mathrm{NaIO}_{4}, D_{\mathrm{h}}$ remains roughly constant up to $X \simeq 40 \%$, after which it rapidly increases, reaching a plateau at $\simeq 1050 \mathrm{~nm}$ for $X \simeq 70 \%$, as schematically represented by the two dashed horizontal lines in Figure 1c. This is in agreement with the plateau observed for the degree of core removal in Figure $1 \mathrm{~b}$ and indicates that the dissolution of the core allows for an increased swelling of the pNIPAM network in the shell. ${ }^{28}$ In particular, this behavior reveals that a critical number of cross-links needs to be cleaved in order to allow the rearrangement of the outer polymer shell. This observation differentiates our study from previous ones in which hollow particles are synthesized by the dissolution of a rigid core and in which much thicker shells are typically obtained. ${ }^{23,29}$ The fitting of the form factors indicates that the cleaved polymer leaves the microgel cores, which is also confirmed by measurement of the dry volume of polymer by atomic force microscopy (AFM) presented later on.

To further support these findings on the core dissolution, we perform a study in which we verify the compatibility of the form factors extracted experimentally at different degradation stages with those calculated from a numerical model for hollow microgels. The model that best reproduces the experimental data at the highest $X$ is determined by adjusting the size of the inner cavity and the monomer density of the polymeric shell in the simulations. A faithful comparison is achieved, as shown in Figure $1 \mathrm{~d}$, for a hole radius that comprises $75 \%$ of the coreshell microgel size (compared to $74 \pm 2 \%$ from the DLS measurements) and an outer shell having an average monomer density of about half that of the standard microgel used for the core particle (see Materials, Models, and Methods and Supporting Information, Figures S12-S15).

Since it is not possible to reproduce the chemical degradation procedure numerically, we mimic this process by taking the hollow model just described, inserting a standard microgel in the central cavity (representing the inner DHEAcross-linked microgel), and subsequently removing a certain number of monomers from the inner core, leading to a decrease of its density (see Materials, Models, and Methods and Figure S16). As shown in Figure 1d, the analysis of the form factors confirms the trend we observe in experiments as a function of $X$, with the first peak of the form factor shifting to smaller wavenumbers and the simultaneous appearance of the third peak in the range of examined wavenumbers. Although we do not explicitly include polydispersity in the simulations, the present model is able to qualitatively reproduce the experimental form factors and, in particular, to capture the relative positions of the peaks detected by SLS.

Temperature Response. Numerical simulations offer us further insights on the internal structure of the microgels upon core degradation by studying their temperature response. In order to substantiate the numerical results with experiments, we perform DLS and SLS measurements below and above the pNIPAM's volume phase transition temperature (VPTT) of 35 ${ }^{\circ} \mathrm{C}$ (Figures S3-S5). At $40{ }^{\circ} \mathrm{C}$, when pNIPAM is in a collapsed state, $D_{\mathrm{h}}$ decreases from $520 \mathrm{~nm}$ (for $X=0 \%$ ) to 490 $\mathrm{nm}$ (for $X \simeq 100 \%$, Figure S3). This indicates that above the VPTT, upon degrading the core particle, the outer shell does not buckle into the particle interior, despite being very thin $(\simeq 15 \mathrm{~nm})$. Nonetheless, the swelling ratio $D_{\mathrm{h}}\left(22{ }^{\circ} \mathrm{C}\right) / D_{\mathrm{h}}(40$ $\left.{ }^{\circ} \mathrm{C}\right)$ for a hollow microgel $(2.1 \pm 0.05)$ is higher than that for 


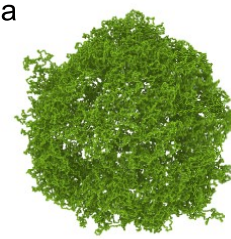

0.0

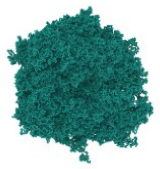

0.65

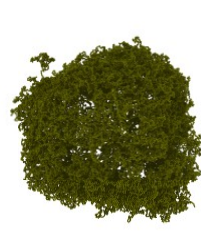

0.35

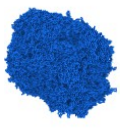

0.8
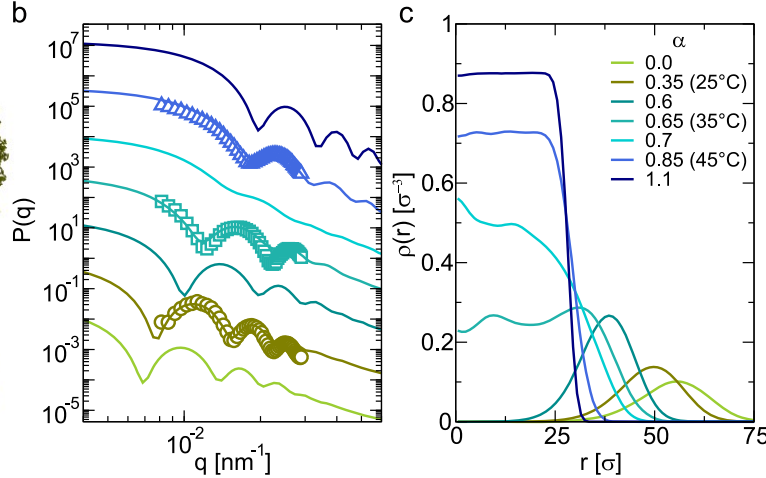

Figure 2. Responsiveness of the hollow microgels across the VPT. (a) Simulation snapshots of hollow microgels as a function of the effective temperature $\alpha$. (b) Experimental (symbols) and numerical (lines) form factors for different effective temperatures. The values of $\alpha=0.35$, 0.65 , and 0.85 correspond to $T=25,35$, and $45{ }^{\circ} \mathrm{C}$, respectively. The comparison is performed by matching the positions of the first peak of $P(q)$, yielding an estimate of the bead size used in the simulations $\sigma=8.25 \mathrm{~nm}$. The form factors are arbitrarily rescaled on the $y$-axis for visual clarity. (c) Density profiles as a function of the distance from the center of mass of the hollow microgel in units of $\sigma$ for different values of the effective temperature $\alpha$.
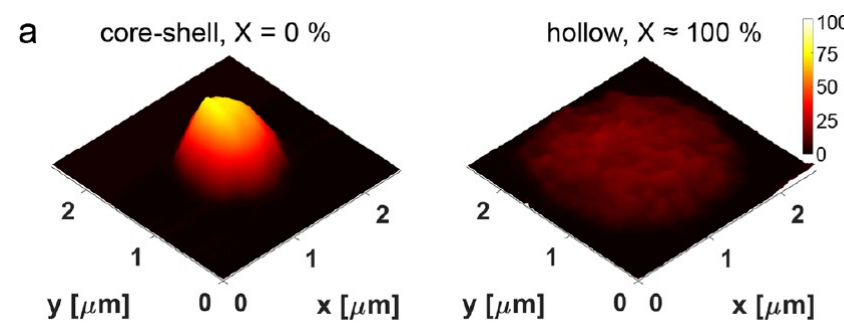

d

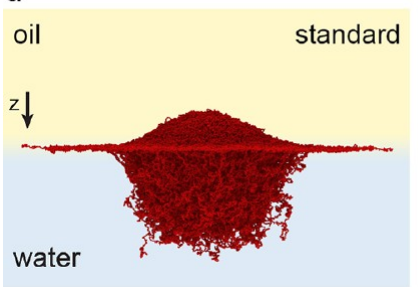

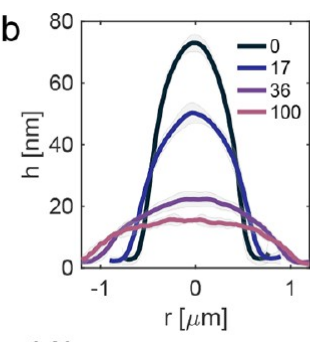

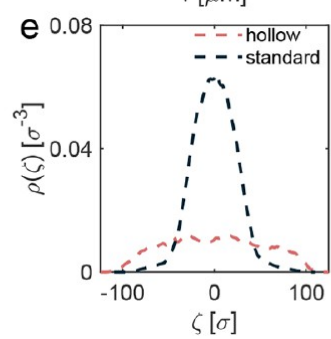

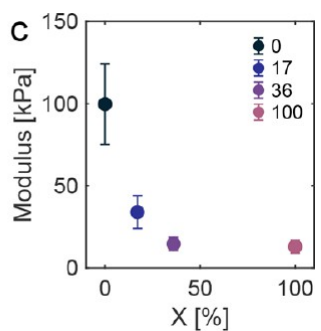

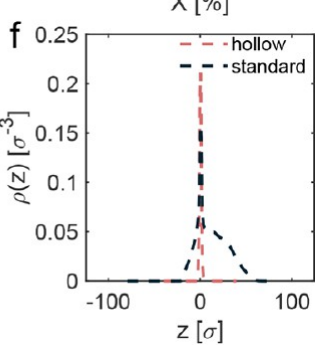

Figure 3. Core-shell and hollow microgels at the liquid interface. (a) 3D profiles of dried core-shell (left) and hollow (right) microgels as obtained by AFM imaging after deposition onto a silicon wafer from the hexane-water interface. (b) Experimental height profiles for different $X(\%)$. The shaded regions correspond to the standard deviations of the height profiles calculated on around 10 particles. (c) Average and standard deviation of the microgels Young's modulus obtained from AFM nanoindentation measurements as a function of $X$. (d) Simulations snapshots of a standard (left) and a hollow (right) microgel adsorbed at a oil-water interface. (e,f) Numerical density profiles on the plane of the interface $\rho(\zeta)$ (e) and across the interface $\rho(z)$ (f) for a hollow microgel and for a standard core-corona microgel; for $\rho(z)$, negative $z$ corresponds to the oil phase.

the initial core-shell particle $(1.66 \pm 0.04)$ because $D_{\mathrm{h}}\left(22^{\circ} \mathrm{C}\right)$ increases with $n \cdot t$ and thus $X$.

The temperature response of the hollow microgels (i.e., with $X \simeq 100 \%$ ) emerging from the SLS measurements is fully captured by the simulations, where the entire range of effective temperatures can be accessed by varying the parameter $\alpha$ of a solvophobic potential (see Materials, Models, and Methods). Figure 2a displays representative simulation snapshots, showing the transition from a fully swollen microgel to a collapsed one at $\alpha=1.1$. It is evident by the snapshots that, due to their intrinsic structure and the reduced density of the shell, these hollow microgels appear more anisotropic and fluffy than the corresponding standard microgels. ${ }^{21}$

The alignment with real units is obtained by matching the first peak of the numerical form factor onto the experimental one at $T=25{ }^{\circ} \mathrm{C}$ and by adopting the same rescaling factor for the other temperatures. ${ }^{21}$ The comparison, reported in Figure $2 \mathrm{~b}$, shows an almost perfect agreement between the form factors of model and laboratory microgels, which is thus realized for $\alpha=0.35,0.65$, and 0.85 corresponding to $T=25$, 35 , and $45{ }^{\circ} \mathrm{C}$, respectively. In particular, we note how the curves progressively shift to higher wavevectors, consistently with the decrease in particle size measured with DLS (Figure S4). Most importantly, we find a crossover at $\alpha=0.7$, roughly corresponding to $36-37{ }^{\circ} \mathrm{C}$, where the form factor has a smoother shape before exhibiting sharp peaks at higher $\alpha$ values.

Having established the correspondence between experimental and numerical form factors, we examine the calculated radial density profiles to confirm the effective formation of hollow microgels below the VPTT. Figure $2 \mathrm{c}$ indeed shows a polymer density profile characterized by a Gaussian-like shell, with a cavity occupying most of the extension of the microgel. However, from around $35^{\circ} \mathrm{C}$, the cavity in the center of the microgel starts to be filled by the polymer chains. Above the VPTT, the hollow particles collapse into a dense globule so 
that the shape of the density profiles resembles those of standard microgels at high temperatures. ${ }^{21}$

Below the VPTT, the volume occupied by the cavity in our hollow microgels corresponds to roughly $40 \%$ of the particle size. This markedly differs from other hollow microgels described in literature, ${ }^{23,29,33-35}$ which are usually obtained by removal of a hard core. In such cases, the size of the cavity is significantly smaller, with a volume occupied by the cavity ranging from $\approx 1.8$ to $6.2 \%$. This has the consequence that, typically, the cavity is also retained at high temperatures, despite a slight size reduction. Indeed, the full shrinkage of the hole is no longer energetically convenient due to the penalty in the elastic bending energy that the thick shell would have to experience. ${ }^{23}$ Therefore, the reported strategy allows for synthesizing hollow microgels, which possess a much thinner shell and a correspondingly larger cavity.

Hollow Microgels at Fluid Interfaces. Individual Microgels. To ultimately rationalize how a given internal polymeric structure of the microgels affects their $2 \mathrm{D}$ assembly at fluid interfaces, we begin by analyzing the conformation that individual particles acquire after adsorption.

An indirect measurement of the 3D shape of the microgels at the oil-water interface is obtained by visualizing the colloids after deposition from the liquid interface onto a solid substrate. AFM imaging allows for a precise quantification of the height profiles of dried microgels. Following previous works, ${ }^{24,36}$ we correlate the profiles measured in this condition with the conformation the microgels had at the interface prior to deposition.

Figure 3a reports typical 3D profiles of isolated microgels deposited on the solid substrate before and after the core degradation process. The reconstructed height profiles at various degrees of core degradation are reported in Figure $3 \mathrm{~b}$. Untreated core-shell microgels (i.e., $X=0 \%$ ) display the characteristically common "core-corona" morphology comprising a denser and thicker core and a more spread-out polymer layer (or corona) of decreasing thickness. ${ }^{22}$ Upon increasing $X$, the height profiles reveal that the microgel flattens and its extension on the interfacial plane increases. In other words, upon removal of the internal polymer network, the microgels become more deformable, and the outer shell, less and less constrained by the reduction of cross-links in the core, can further stretch and expand at the interface. Ultimately, a totally different profile is obtained for the hollow particle $(X \simeq 100 \%)$, which takes up a flat disk-like shape at the interface, with a maximum thickness of around $15 \mathrm{~nm}$ after deposition. In all cases, an external corona composed by the outermost polymer chains, which spread out on the interfacial plane, is found around the microgels, as it is clearly shown by AFM phase images (Figure S6 and Figure 5a).

This conformation differs from others reported in literature, where hollow particles with a thicker shell with respect to the ones investigated here preserve a small cavity even when absorbed at the interface. ${ }^{23}$ Instead, it may resemble at a first sight that of ultralow cross-linked (ULC) microgels, which are synthesized in the absence of cross-linkers. ${ }^{37,38}$ However, although a flattening of the particle is observed for both our hollow microgels and ULC microgels, in the present case, it appears to be much more pronounced with respect to what was observed, for instance, in ref 37.

Furthermore, the change in morphology observed during core degradation is different from the effect of reducing the cross-linker concentration in standard batch-synthesized microgels. For the latter particles, decreasing the internal cross-linking density also results in an increased spreading at the interface together with a concomitant decrease of the maximum height. ${ }^{39,40}$ However, in that case, the height profile retains a Gaussian-like shape, which remains overall qualitatively similar for different cross-linking degrees. ${ }^{24}$

A closer look at the height profiles for our microgels reveals that their maximum height decreases up to 3 times the initial height already for only $\approx 40 \%$ of core removal, suggesting that, for $X \gtrsim 40 \%$, a critical number of cross-links in the internal polymer network are cleaved and the particle stiffness rapidly decreases.

A more direct measure of the mechanical properties of the microgels is obtained by AFM nanoindentation experiments, measured in water after particle deposition from the oil-water interface (see Materials, Models, and Methods). The data in Figure 3c, complemented by Figure S8, display a marked reduction of the particles' Young's modulus, which correlates well with the change in morphology. For $X \approx 40 \%$, the particles have lost most of their internal stiffness and can consequently spread more at the interface under the action of the interfacial tension. Finally, the height profiles also indicate that the core degradation is associated with a loss of polymer; that is, the cleaved polymers leave the inner part of the particle. As the dry height profile of the microgels is a direct representation of polymer mass distribution, their integral represents the particle total dry mass. In Figure S7, we show that the evolution of the height profiles corresponds to a mass loss, again correlated to the decrease in particle stiffness.

In simulations, we compare the particle morphology of a standard and a hollow microgel. Simulation snapshots are reported in Figure 3d and Figure S9 and immediately illustrate the difference between the two architectures. The microgel density profiles projected onto the plane of the interface $\rho(\zeta)$ (see Materials, Models, and Methods for its definition), reported in Figure 3e, closely mirror the experimentally measured ones after deposition (Figure 3b). In particular, these confirm a similar distribution of the polymer network between core-shell particles and standard microgels, whereas for the hollow ones, the numerically extracted shape compares well to that obtained experimentally. In Figure $3 \mathrm{f}$, we also report the profiles across the interface $\rho(z)$. Here, as opposed to the case of the standard microgel, which shows different protrusions in oil and water, the hollow microgel is essentially confined within the interface where it forms a uniform thin layer, barely protruding into either fluid. A comparison between simulations and experiments shows that the extension of the microgel across the interface obtained from simulations is about few $\sigma$, comparing quite well with the experimental height in Figure $3 b$.

Finally, we characterize the extent of the in-plane deformation at the oil-water interface relative to the bulk size by the ratio $D_{\mathrm{i}}^{0} / D_{\mathrm{h}}$, where $D_{\mathrm{i}}^{0}$ is the particle diameter at the interface as measured by AFM phase images or extracted from the simulations. The core-shell microgels have a stretching ratio of $1.7 \pm 0.1$, whereas the hollow microgels reach a value of $2.4 \pm 0.1$, following the same trend of the values extracted from the numerical simulations (1.4 and 1.9, respectively).

2D Assembly and Response upon Compression. After examining their single-particle conformation at the oil-water interface, we study $2 \mathrm{D}$ assemblies of the hollow microgels to link their architecture with their $2 \mathrm{D}$ phase behavior and response upon interfacial compression. To this purpose, we 


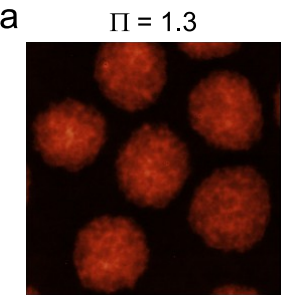

b

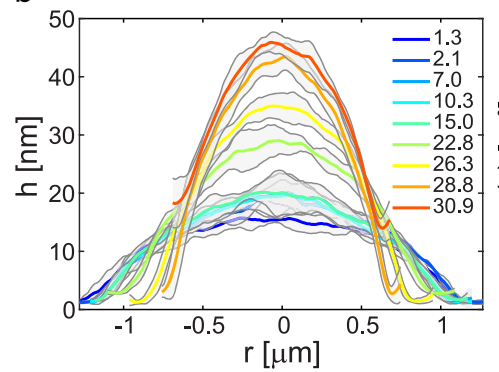

$\Pi=7.0$

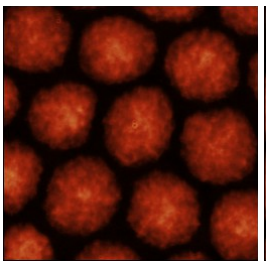

C

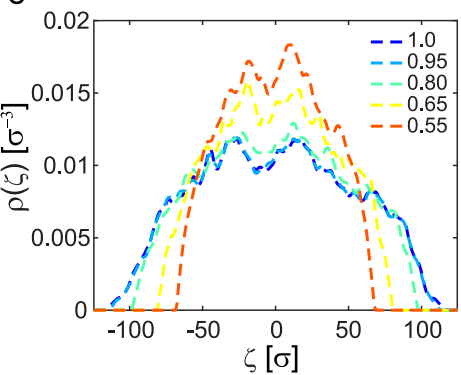

$\Pi=15.0$
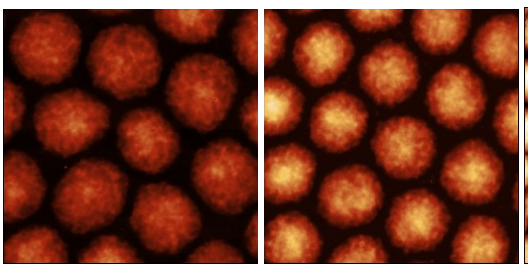

d

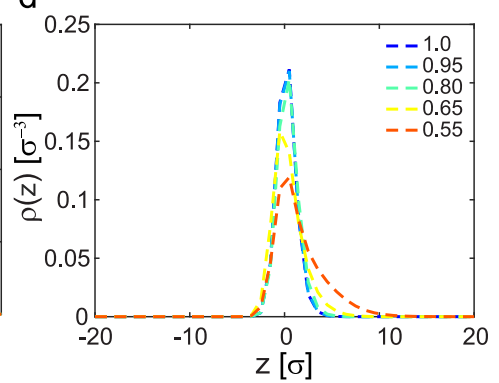

Figure 4. Compression of hollow microgels at the liquid interface. (a) AFM height images showing the monolayer microstructure at increasing surface pressure $\Pi$. Scale bar: $2 \mu \mathrm{m}$. (b) Microgel height profiles extracted from images as in (a) at increasing $\Pi$. The shaded regions correspond to the standard deviations of the height profiles calculated on around 6-10 particles. (c,d) Numerical density profiles within the plane of the interface $\rho(\zeta)(\mathrm{c})$ and across the interface $\rho(z)$ (d) for different degrees of radial compression of the hollow microgels defined as $D_{\mathrm{i}} / D_{\mathrm{i}}^{0}$, where $D_{\mathrm{i}}^{0}$ is the interfacial size of hollow particle without compression; for $\rho(z)$, negative $z$ corresponds to the oil phase.

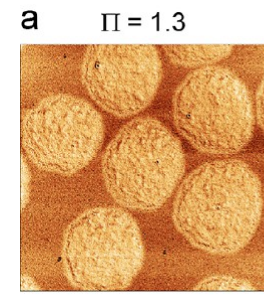

$\Pi=22.8$

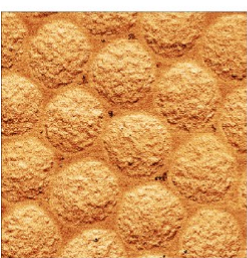

$\Pi=7.0$

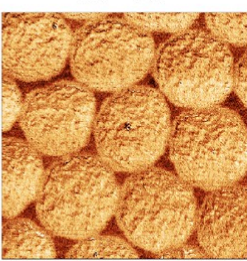

$\Pi=26.3$

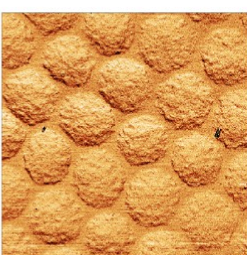

$\Pi=15.0$

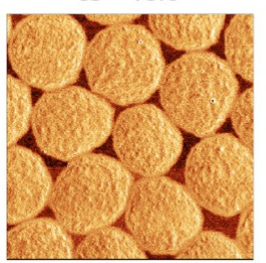

$\Pi=30.9$

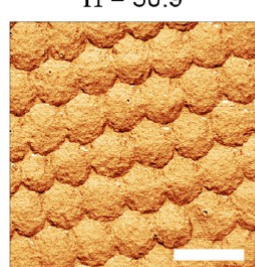

b

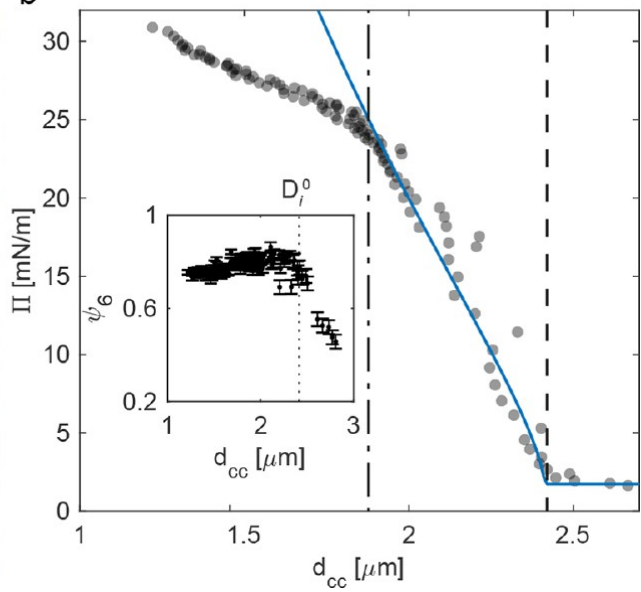

Figure 5. Collective behavior of hollow microgels at the oil-water interface. (a) AFM phase images showing the microgels' conformation at different values of the surface pressure $\Pi$. Scale bar: $2 \mu \mathrm{m}$. (b) Compression isotherms reporting the surface pressure $\Pi$ versus the center-tocenter distance $d_{\mathrm{cc}}$ in the particle monolayer. The dashed lines separate the three different regimes (see main text). The first part of the compression curve is fitted to eq 5. Inset: hexatic order parameter $\psi_{6}$ as a function of $d_{\mathrm{cc}}$. The black dotted line indicates the diameter of a single particle at the liquid interface prior to compression $\left(D_{\mathrm{i}}^{0}\right)$.

spread microgels at a hexane-water interface in a custom-built Langmuir trough and transfer monolayers onto a solid substrate under continuous compression, as detailed in the Materials, Models, and Methods section.

Figure 4 reports the microgels' conformation within a monolayer upon increasing compression of the liquid interface, as revealed by AFM height images. At low pressures (up to $\Pi$ $\approx 15 \mathrm{mN} \cdot \mathrm{m}^{-1}$ ), the microgels deform in a limited manner (see height profiles in Figure $4 \mathrm{~b}$ ): both the cross-sectional area within the interface plane and the height of each microgel remain approximately constant. In this compression range, microgels organize into a hexagonal assembly and show no appreciable deformation. We hypothesize that increasing compression in this regime mostly affects the outermost polymer chains, which occupy all the available space between the particles (see discussion below). These observations are also captured by the numerical simulations results reported in Figure 4c,d. Here, the radial compression is progressively carried out on single hollow microgels (see Materials, Models, and Methods). The numerical density profiles, both on the plane of the interface (Figure 4c) and across the interface (Figure 4d), are essentially not affected if $D_{\mathrm{i}} / D_{\mathrm{i}}^{0} \lesssim 5 \%$ (corresponding to $\Pi \lesssim 15 \mathrm{mN} \cdot \mathrm{m}^{-1}$ in the experiments), resembling in all respects the profile of an uncompressed particle. However, at higher $\Pi$, the rearrangement of the polymeric network involves the whole microgel. For $\Pi>20$ $\mathrm{mN} \cdot \mathrm{m}^{-1}$, the hollow microgels significantly deform to accommodate the increased pressure: the cross-sectional area decreases, while the height of the deposited microgels increases (Figure $4 \mathrm{~b}$ ). At this level of compression, part of the polymer chains desorb from the interface and the particle expands in the third dimension, perpendicularly to the interface. This 
extension in the third dimension is clearly visible in the $\rho(z)$ profiles of the simulated microgels reported in Figure $4 \mathrm{~d}$. Furthermore, as more clearly evidenced from phase images shown in Figure 5a, in this regime, the particles also deform within the interface plane, with the outer polymer chains that fill the voids between the particles' cores to fully occupy the available space at the interface.

Information on the macroscopic response of the microgel monolayer comes from the compression curve reported in Figure $5 b$, which displays the surface pressure as a function of the microgel-microgel interparticle distance $d_{c c}$. It can be noted that the curve presents three different slopes, depending on the range of $d_{\mathrm{cc}}$ analyzed. The first regime at very low and almost constant surface pressure $\left(d_{\mathrm{cc}}>2.4 \mu \mathrm{m}\right)$ corresponds to a dilute monolayer, where the distance between particles is higher than the size of a single isolated particle at the interface, and the result is a disordered arrangement (Figure $5 \mathrm{a}, \Pi=1.3$ $\left.\mathrm{mN} \cdot \mathrm{m}^{-1}\right)$. Compressing such a dilute monolayer causes a progressive increase of the effective concentration of microgels at the interface until all particles in the assembly are in contact through their outer polymeric chains. This happens when the distance between microgels is approximately equal to their lateral size at the interface, marked in Figure $5 \mathrm{~b}$ as $D_{\mathrm{i}}^{0}$. Correspondingly, the particles assemble into a hexagonal packing, as evidenced by the gradual increase in the hexagonal order parameter $\psi_{6}$, which reaches a maximum at around $d_{\mathrm{cc}}=$ $2.2 \mu \mathrm{m}$ (inset in Figure 5b, corresponding AFM image in Figure 4a and Figure S10).

Upon increasing compression, the monolayer enters a second regime where the surface pressure rises while the particle morphology does not exhibit significant changes, as previously described. Correspondingly, the monolayer retains a high degree of hexagonal order, and $\psi_{6}$ substantially remains constant. We note that the slight decrease of $\psi_{6}$ is presumably due to both size and mechanical polydispersity, which becomes relevant when microgels are extensively compressed with respect to their initial conformation, as previously observed in ref 41 The effective mechanical behavior of the monolayer for small particle deformations is captured by assuming that the microgels interact via a generalized Hertzian potential (see Materials, Models, and Methods). From this description, we extract two important facts. First, the fit nicely describes the data only for values of $d_{c c}$ for which the height profiles of the microgels do not exhibit significant deformation in the third dimension. Second, the fit gives a power-law exponent $a=1.7$ \pm 0.1 , which is close to the one obtained for common corecorona microgels at the hexane-water interface $(a=1.8 \pm$ 0.2). ${ }^{17}$ These two facts indicate that the macroscopic response of the monolayer in this compression range is similar, irrespective of the internal microgel structure. As a conclusion, we argue that only the outer pNIPAM chains determine the mechanical behavior upon compression in this surface pressure range.

Finally, we observe a third regime in the compression curve. For $d_{\mathrm{cc}}<2 \mu \mathrm{m}$ and $\Pi>20 \mathrm{mN} \cdot \mathrm{m}^{-1}$, the out-of-plane deformation of the microgels translates into a variation of the slope of the compression isotherm. In this regime, when microgels completely cover the fluid interface, a further increase in the surface pressure can be explained in terms of interfacial elasticity. ${ }^{42}$ At first sight, the slope change reminds one of similar trends that have been reported for batchsynthesized microgels. ${ }^{41,43}$ However, in the latter case, for microgels of similar size, this is typically ascribed to an isostructural solid-solid phase transition which occurs when the outer microgel coronas collapse, enabling core-core contacts. $^{43}$ In the case of hollow microgels instead, the absence of polymer in the core allows the particles to shrink in the interfacial plane without affecting their hexagonal packing. On the macroscopic level, this results in a $2 \mathrm{D}$ assembly that maintains the same hexagonal order throughout the compression isotherm but with a continuously decreasing particle size and, correspondingly, lattice constant (Figure S11).

\section{CONCLUSIONS}

In this work, we coupled experiments and numerical simulations to provide a detailed description of the internal polymer distribution of core-shell microgels undergoing varying degrees of core degradation. In particular, by endowing the soft cores with chemically cleavable cross-linkers, we obtained particles with controlled internal structures, ranging from the as-synthesized core-shell case to hollow microgels with a thin shell $\left(\sim 115 \mathrm{~nm}\right.$ at $\left.22{ }^{\circ} \mathrm{C}\right)$ and a large cavity $(\sim 640$ $\mathrm{nm}$ ) after full degradation of the core. Such architectures, encompassing small ratios of polymer shell thickness versus size of the cavity, are not easily accessible by common synthetic strategies, where hollow particles are made by dissolving a hard inorganic core. ${ }^{23,32}$ In the latter case, the core does not deform in aqueous suspension, and thicker shells are produced, causing the hollow microgels to behave similarly to their core-shell parent particles, with comparable swelling and deswelling properties. $^{29,34}$

We found that the internal structure of the particles, which we characterize in the bulk aqueous phase, is tightly linked to both the global mechanical response of microgel monolayers at a fluid interface and their microstructure upon isothermal compression. In particular, we rationalized the properties of the two-dimensional assemblies by characterizing the morphology of individual microgels at the fluid interface. Here, we evidenced that the monolayer response at low compression is similar for all microgel internal structures and appears to be solely governed by the rearrangements of the outer polymer chains constituting the microgel corona at the interface. Conversely, a greater degree of compression led to a different phase behavior of the hollow microgels compared to that of common batch-synthesized ones, with a continuous compaction of a hexagonally packed monolayer instead of the commonly occurring isostructural transition found for standard microgels of comparable size. ${ }^{43}$ Similar continuous transitions have been already reported in the case of nanometer-sized microgels ${ }^{41}$ or for microgels with a low cross-linking density. ${ }^{39,40}$ However, in these cases, the hexatic order usually drops upon increasing compression, leading to a disordered monolayer at high pressure. The absence of a core instead enables the hollow microgels to expand out of the interface plane, allowing the assembly to maintain a high degree of hexatic order throughout the compression isotherm. Incidentally, the behavior observed for bulk suspensions of hollow microgels is radically different from that described here at the interface, indicating a central role of the surface tension in determining their collective behavior. Indeed, a recent study has shown the absence of crystal formation in three dimensions in favor of deswelling, interpenetration, and faceting depending on the concentration regime under investigation. ${ }^{44}$

Our findings provide a comprehensive understanding on the effect of the internal structure on the interfacial behavior of soft 
colloids. In particular, we remark the fact that the structural and mechanical behavior at the fluid interface under low compressions, i.e., corresponding to small overlaps between the particles, appears to be independent of the presence of an inner cavity. This presents us with new challenges and opportunities during synthesis. Indeed, most synthetic efforts are targeted to control the radial cross-linking density profiles of the microgels. However, the interfacial response is initially governed by the properties of a corona of loosely cross-linked or even un-cross-linked chains that surround the microgel within the interface plane. Based on these considerations, we expect that a bigger impact in the tailoring of the monolayer response can be achieved by engineering the properties of the coronas, rather than of the overall density profiles. Possible strategies encompass the controlled growth of shells of linear polymers of different length after completing the cross-linking reaction. At the same time, a closer inspection of the fate of the chains composing the corona during compression would certainly greatly add to our understanding. Nonetheless, the importance of the internal structure emerges at higher compression, delineating a large, and only partially explored, parameter space for the controlled designed of tailored twodimensional assemblies of soft particles.

\section{MATERIALS, MODELS, AND METHODS}

Synthesis of Core-Shell and Hollow Microgels. The hollow microgels used in this study were synthesized by a two-step freeradical precipitation polymerization following, with some modifications, a procedure previously reported by Nayak et al. ${ }^{28}$

Core Preparation. N-Isopropylacrylamide (NIPAM, $0.5 \mathrm{~g}$ ), $5 \mathrm{~mol}$ $\%$ of methacrylic acid (MAA), and $10 \mathrm{~mol} \%$ of $N, N^{\prime}-(1,2-$ dihydroxyethylene)bis(acrylamide) (DHEA) were dissolved in 50 $\mathrm{mL}$ of MQ water at room temperature. The reaction mixture was then immersed into an oil bath at $80{ }^{\circ} \mathrm{C}$ and purged with nitrogen for $1 \mathrm{~h}$. The reaction was started by adding $6.5 \mathrm{mg}$ of potassium persulfate (KPS) previously dissolved in $1 \mathrm{~mL}$ of MQ water and purged with nitrogen. The polymerization was carried out for $6 \mathrm{~h}$ in a sealed flask. Afterward, the colloidal suspension was cleaned by dialysis for 1 week and by eight centrifugation cycles and resuspension in pure water. The use of a lower amount of initiator and the absence of surfactants in the reaction mixture allowed us to produce microgels of bigger size in comparison to those in ref 28.

Shell Addition. In a reaction flask, $29 \mathrm{mg}$ of the core microgels was dispersed in $10 \mathrm{~mL}$ of MQ water at $80{ }^{\circ} \mathrm{C}$ and purged with nitrogen for $1 \mathrm{~h}$. In parallel, NIPAM $(0.1 \mathrm{~g}), 5 \mathrm{~mol} \%$ of MAA, and $5 \mathrm{~mol} \%$ of $N, N^{\prime}$-methylenebis(acrylamide) (BIS) were dissolved in $10 \mathrm{~mL}$ of MQ water at room temperature and purged with nitrogen for $1 \mathrm{~h}$. In a third vial, $2 \mathrm{mg}$ of KPS was dissolved in $1 \mathrm{~mL}$ of MQ water and purged with nitrogen. Still keeping the flask sealed, the temperature of the solution containing the core particles was increased to $80{ }^{\circ} \mathrm{C}$ prior to adding the initiator. Immediately afterward, we started the feeding of the monomer solution (at $166 \mu \mathrm{L} / \mathrm{min}$ ) into the reaction flask. When the feeding was terminated, the reaction was quenched by opening the flask in air and placing it in an ice bath. The obtained colloidal suspension was cleaned by dialysis for 1 week and by eight centrifugation cycles and resuspension in pure water. A polymerization reaction by continuous monomer addition was chosen over the more common batch reaction in order to ensure a more controlled and homogeneous shell growth. ${ }^{25,26}$ The final shell is therefore chemically grafted onto the surface of the polymeric cores. Additionally, the fact that the core microgels are collapsed during synthesis imposes that there is only limited diffusion of BIS molecules inside the polymeric core, leading to a small interfacial region where an interpenetrated network is formed. ${ }^{28}$

Core Degradation. The controlled degradation of the particles' cores was achieved by exposing core-shell particles to known amounts of sodium periodate $\left(\mathrm{NaIO}_{4}\right)$ over different times. In particular, the required amount of mols of sodium periodate with respect to DHEA molecules in solution was added to $500 \mu \mathrm{L}$ of microgel suspension, and the total volume was increased to $1.5 \mathrm{~mL}$. The amount of DHEA was estimated knowing the microgel concentration, the cores' size, and assuming that the entire amount of DHEA added during the cores' synthesis was incorporated in the particles. Three different exposure times were used: $20 \mathrm{~h}, 3$ days, and 8 days. When the required time elapsed, the particle suspension was cleaned by centrifugation and supernatant exchanged once a day for 10 days in order to ensure complete removal of loose polymer chains from the interior of the particles.

Experimental Methods. DLS and SLS. Dynamic light scattering experiments were performed using a Zetasizer (Malvern, UK). The scattering vector for DLS experiments was $q=0.026 \mathrm{~nm}^{-1}$. The samples were let to equilibrate for $15 \mathrm{~min}$ at the required temperature $\left(22\right.$ or $40{ }^{\circ} \mathrm{C}$ ) prior to performing six consecutive measurements. To record volume phase transition curves, the temperature was scanned from 20 to $50{ }^{\circ} \mathrm{C}$ with $2{ }^{\circ} \mathrm{C}$ steps. At each temperature, the sample was left to equilibrate for $10 \mathrm{~min}$ before four consecutive measurements were performed. For SLS, a CGS-3 compact goniometer (ALV, Germany) system was used, equipped with a Nd:YAG laser, $\lambda=532 \mathrm{~nm}$, output power of $50 \mathrm{~mW}$ before optical isolator, with measuring angles from 30 to $150^{\circ}$ with $2^{\circ}$ steps.

Form Factor Fitting Procedure. Static scattering form factor analysis was performed using the FitIt! tool developed by Otto Virtanen for MATLAB. ${ }^{45}$ The intensity distributions were fitted by assuming the following density profile:

$$
\rho(r)=\left\{\begin{array}{l}
\rho_{0} \mathrm{e}^{\lambda(r-R)}, 0 \leq r \leq R \\
0, r>R
\end{array}\right.
$$

This minimal model gives an excellent description of the experimental form factors of the hollow microgels (see Figure S1) with only two parameters: the size $R$ and the rate of decay of the density from the outer surface toward the center of the microgel $\lambda$. The size of the microgels is assumed to be normally distributed.

To calculate the mass removed during the reaction with $\mathrm{NaIO}_{4}$ : $\Delta M=M_{0}-M$, we calculated the final mass as $M=m \int_{0}^{R} 4 \pi r^{2} \rho(r) \mathrm{d} r$ and the initial mass as $M_{0}=4 / 3 \pi R^{3} m \rho_{0}$, where $m$ is the mass of a monomer unit. The relative mass loss $\frac{\Delta M}{M_{0}}$ is thus calculated as

$$
\frac{\Delta M}{M_{0}}=1-3 \frac{\lambda R(\lambda R-2)+2\left(1-\mathrm{e}^{-\lambda R}\right)}{\lambda^{3} R^{3}}
$$

We then estimated the relative mass loss of the core $X$ as

$$
\frac{M_{\mathrm{c} 0}-M_{\mathrm{c}}}{M_{\mathrm{c} 0}} \simeq \frac{\Delta M}{M_{0}}\left(\frac{M_{0}}{M_{\mathrm{c} 0}}\right)=\frac{\Delta M}{M_{0}}\left(\frac{R^{3}}{R_{\mathrm{c}}^{3}}\right)
$$

where $M_{c}$ and $M_{c 0}$ are the final and the initial mass of the core, and $R_{c}$ is the radius of the core. We do not expect the uncertainty in $R$ and $\lambda$ to affect the relative molar mass estimation. In fact, $R$ depends on the distance between the minima in the scattering intensity or, in other words, on its periodicity. $\lambda$ depends instead on the relative magnitude of the local maxima that is on the rate of decay of the signal. Since the size of the microgels in question is about $1 \mu \mathrm{m}$, we do not expect minima below $q \approx 0.01 \mathrm{~nm}^{-1}$. Therefore, the low scattering vectors contain little (or even no) information about the magnitude of $R$, which is instead well-captured by the distance between the minima for $q>0.01 \mathrm{~nm}^{-1}$. Analogously, $\lambda$ mainly depends on the relative magnitude of the local maxima for $q \gg 0.01 \mathrm{~nm}^{-1}$. Overall, the uncertainty in $R$ and $\lambda$ depends on the scattering vectors and intensity at the minima and maxima for $q \geq 0.01 \mathrm{~nm}^{-1}$, which is the region covered by our measurements.

The $95 \%$ confidence intervals of $X$ were estimated using the Monte Carlo method. In particular, the lower and upper bounds were constructed by calculating the 5 th and the 95 th percentile of the distribution of $X$ obtained by propagating the uncertainty in $R, R_{\mathcal{O}}$ and 
$\lambda$ after 10000 trials. The values of $X$ plotted in Figure 1 as symbols are the medians of such distributions.

Deposition of Isolated Microgels from a Hexane-Water Interface. Microgels were deposited from a hexane-water interface onto silicon wafers for atomic force microscopy imaging of isolated dried particles following an already reported procedure. ${ }^{46}$ Silicon wafers were cut into pieces and cleaned by $15 \mathrm{~min}$ ultrasonication in toluene (Fluka Analytical, 99.7\%), isopropyl alcohol (Fisher Chemical, 99.97\%), acetone, ethanol, and MQ water. A piece of silicon wafer was placed inside a Teflon beaker on the arm of a linear motion driver and immersed in water. Successively, a liquid interface was created between MQ water and $n$-hexane (Sigma-Aldrich, HPLC grade $95 \%$ ). Around $100 \mu \mathrm{L}$ of the microgel suspension was injected at the interface after appropriate dilution in a 4:1 MQwater/isopropyl alcohol solution. After $10 \mathrm{~min}$ equilibration time, extraction of the substrate was conducted at a speed of $25 \mu \mathrm{m} \cdot \mathrm{s}^{-1}$ to collect the microgels by sweeping through the liquid interface.

Langmuir Trough Deposition. Microgels self-assembled at the hexane-water interface at controlled surface pressure $(\Pi)$ values were deposited onto silicon wafers for visualization using a custom-made setup already reported in the literature. ${ }^{43}$ We used a KSV5000 Langmuir trough equipped with a dipper arm immersed in water for holding a silicon substrate forming an angle of approximately $30^{\circ}$ with the water surface. The silicon substrate was further cleaned in a UVOzone cleaner (UV/Ozone Procleaner Plus, Bioforce Nanosciences) for $15 \mathrm{~min}$ to ensure a hydrophilic surface prior to microgel deposition. After a hexane-water interface was formed, the substrate was lifted to pierce the liquid interface. Microgels were then injected on the liquid interface, while the surface pressure was simultaneously measured with a platinum Wilhelmy plate. When the required initial surface pressure was reached, the injection was stopped and the interface was left to equilibrate for $15 \mathrm{~min}$. Successively, the dipper was activated to extract the substrate at a constant speed of $0.3 \mathrm{~mm}$. $\mathrm{min}^{-1}$, and after $2 \mathrm{~min}$, the barriers started moving at a compression speed of $2.3 \mathrm{~mm} \cdot \mathrm{min}^{-1}$. When the compression finished, the barriers were immediately opened while the substrate was still moving up in order to achieve a discontinuity in microgel concentration deposited on the silicon wafer.

The conformation of microgels at the interface and their 2D assembly as a function of the surface pressure was then inferred by analyzing the substrates using AFM. Images from the initial position of the three-phase contact line to the end of the substrate were recorded at a fixed distance of $500 \mu \mathrm{m}$. The discontinuity in microgel deposition ensures a correct assignment of the surface pressure value measured at the liquid interface during compression to the corresponding position on the silicon substrate. More specifically, the highest value of surface pressure measured during the experiment was assigned to the position on the substrate corresponding to the highest density of microgels. Consequently, knowing the dipper speed and the distance between AFM images of the substrate, we scanned the surface pressure curve backward, assigning to each AFM image its corresponding value of $\Pi$.

AFM Imaging and Analysis. Microgels deposited on silicon wafers were characterized by AFM (Bruker Icon Dimension), in tapping mode, using cantilevers with $\sim 300 \mathrm{kHz}$ resonance frequency and a $\sim 26 \mathrm{~N} \cdot \mathrm{m}^{-1}$ spring constant (OMCLAC160TS-R3, Olympus). Height and phase images were recorded at the same time. Images were first processed with Gwyddion and successively analyzed with custom MATLAB codes. The following procedure was used to obtain an average height profile: for each microgel, horizontal and vertical profiles passing through its center were measured from AFM height images. Successively, an average of over around 10 microgels was obtained by aligning each profile by its center value. For the height profiles of a single microgel inside $2 \mathrm{D}$ assemblies (Figure 4 ), the same procedure was used; the profiles were then cut on the $x$-axis to exclude neighboring microgels.

The average interparticle distance $d_{\mathrm{cc}}$ at different $\Pi$ was estimated by extracting the positions of the microgels from AFM images taken at different locations on the substrates. For a given set of particles' coordinates $(x, y), d_{\mathrm{cc}}$ was calculated as the average distance between neighboring particles. The neighbors' list was constructed based on the Voronoi tessellation using the Freud open-source Python libraries. ${ }^{47}$ Such a neighbors' list was also used to calculate the average hexatic order parameter parameter $\psi_{6}$ :

$$
\psi_{6}=\left\langle\frac{1}{N_{j}} \sum_{k=1}^{N_{j}} \mathrm{e}^{i 6 \theta_{j k}}\right\rangle
$$

where $N_{j}$ is the number of neighbors of the $j$ th particle in the AFM image, $\theta_{j k}$ is the angle between the unit vector $(1,0)$, and the vector $\mathbf{r}$ $=\mathbf{r}_{k}-\mathbf{r}_{j}$ connecting particle $j$ and its $k$ th neighbor.

The compression isotherms $\left(\Pi=f\left(d_{\mathrm{cc}}\right)\right)$ were fitted by assuming the following: (i) the interparticle interactions between microgels adsorbed at the oil-water interface can be described by the generalized Hertzian potential ${ }^{48,49}$ of the form

$$
U(r)=\frac{\epsilon}{a}\left(1-\frac{r}{s}\right)^{a} \Theta\left(1-\frac{r}{s}\right)
$$

where $\epsilon$ is the energy scale, $s$ is the diameter of an isolated microgel at the interface, $\Theta$ is the Heaviside step function, and $a$ is a power-law exponent that defines the softness of the potential $(a=5 / 2$ for the purely Hertzian case); (ii) the microgels assemble into a hexagonal lattice: ${ }^{17}$

$$
\left.\Pi\right|_{T=0}=-\frac{\partial E_{\text {hex }}}{\partial A_{\text {hex }}}=\frac{\epsilon \sqrt{3}}{a s r}\left(1-\frac{r}{a}\right)^{a-1} \Theta\left(1-\frac{r}{s}\right)
$$

The fitting was performed using the curve fitting tool of MATLAB. A good description of the experimental data was obtained by constraining the range of $d_{\mathrm{cc}}$ to $0.75-1 \mathrm{~s}$. This is because for $d_{\mathrm{cc}} \geq$ $s$ the monolayers are not hexagonally packed, and for $d_{\mathrm{cc}} \lesssim 0.75 \mathrm{~s}, \Pi$ displays a sudden change of slope that cannot be captured by the functional form of the generalized Hertzian potential.

Nanoindentation. Nanoindentation measurements were performed in MQ water using an Asylum Research MFP 3D AFM (Oxford Instruments, Santa Barbara, CA) on microgels deposited from the hexane-water interface onto silicon wafers. A silica microparticle (diameter $=2 \mu \mathrm{m}$, Microparticles $\mathrm{GmbH}$ ) was glued with a two-component epoxy glue (UHU Plus endfest, UHU GmbH, Germany) to the end of a tipless cantilever using a home-built micromanipulator. The normal spring constant $\left(0.14 \mathrm{~N} \cdot \mathrm{m}^{-1}\right)$ of the Au-coated cantilever (CSC-38, Mikromash, Bulgaria) was measured by the thermal-noise method. ${ }^{50}$ Force versus distance curves were recorded using the force-mapping mode over an area of $10 \times 10 \mu \mathrm{m}^{2}$. The applied force was kept smaller than $2 \mathrm{nN}$ to reduce any substrate effect. Force versus distance curves recorded at the center of the microgels were then converted into force versus indentation curves (the indentation was measured by subtracting the cantilever deflection by the vertical piezodisplacement) and fitted using the Hertz model:

$$
F=\frac{4 \sqrt{R}}{3\left(1-\nu^{2}\right)} E \delta^{1.5}
$$

where $F$ is the applied force and $R$ is the radius of the colloid used. By knowing the silica probe elastic modulus and the sample Poisson's ratio ( $\nu$ ), Young's modulus $E$ of the microgels was obtained by applying the Hertz fit function in the dedicated Asylum Research software (version AR13).

Numerical Models and Methods. In Silico Synthesis. The coarse-grained microgels used for the simulations are characterized by a fully bonded, disordered network, as previously described. ${ }^{21,51}$ This is obtained starting from an ensemble of $N$ two- and four-folded patchy particles, which mimic the connectivity of monomers and cross-linkers employed in the chemical synthesis. The spherical shape of the microgels is obtained by letting the patchy particles selfassemble in a spherical cavity of radius $Z$. For the numerical synthesis of hollow microgels, we also apply an inner spherical force field which does not allow patchy particles to enter a region of radius $Z_{\text {in }}$, with $Z_{\text {in }}$ $<Z$. For standard microgels, we add a designing force on cross-linkers only in order to reproduce their inhomogeneous distribution between 
core and outer periphery as detailed in ref 21 . For the hollow microgels investigated here, there are no major differences in applying or not the additional designing force (Figure S10). Each microgel is assembled with the OXDNA simulation package. ${ }^{52}$

The assembly process is terminated once almost all possible bonds are formed (>99.9\%). Subsequently, the topology of the microgel is fixed by means of the Kremer-Grest bead spring model, ${ }^{53}$ according to which all particles experience a steric repulsion via the WeeksChandler-Anderson (WCA) potential,

$$
V_{\mathrm{WCA}}(r)= \begin{cases}4 \epsilon\left[\left(\frac{\sigma}{r}\right)^{12}-\left(\frac{\sigma}{r}\right)^{6}\right]+\epsilon & \text { if } r \leq 2^{1 / 6} \sigma \\ 0 & \text { otherwise. }\end{cases}
$$

As commonly defined in coarse-grained simulations, $\epsilon$ sets the energy scale, $\sigma$ is the diameter of the particles, which also defines the unit of length, and $r$ is the distance between two particles. Also, bonded beads interact via the finitely extensible nonlinear elastic (FENE) potential:

$$
V_{\mathrm{FENE}}(r)=-\epsilon k_{\mathrm{F}} R_{0}^{2} \ln \left[1-\left(\frac{r}{R_{0} \sigma}\right)^{2}\right] \text { if } r<R_{0} \sigma
$$

with $k_{\mathrm{F}}=15$, which determines the stiffness of the bond, and $R_{0}=1.5$ is the maximum bond distance. In this work, we study microgels with $Z=100 \sigma$ and $75 \sigma$ with a fraction of cross-linker $c=5 \%$. The model for standard microgels employed in this work is the one described in ref 21, with an average internal density of monomers $\rho \sim 0.08 \sigma^{-3}$. For the hollow particles, we employ $Z_{\text {in }}=0.75 Z$ and $\rho=0.035 \sigma^{-3}$; the consequences of varying these parameters are discussed in the Supporting Information, Figures S12-S15. In all cases, we assume that the low amount of charges present after the chemical synthesis does not affect the structural features and the swelling behavior of the in silico microgels.

The protocol just described does not allow one to reproduce all of the stages of the chemical synthesis procedure. Nonetheless, it allows generating standard and hollow microgels independently, ensuring that the structural features of the final particles resemble experimental results. For this reason, in order to assess the core degradation process, we also make use of a standard microgel with $Z=90 \sigma, \rho=$ $0.08 \sigma^{-3}$, and $c=10 \%$ (corresponding to the DHEA-cross-linked microgel in the chemical synthesis), which is then inserted in the central cavity of a hollow microgel, mimicking a core-shell particle. By progressively removing the internal monomers and thus reducing the internal density, we reproduce the experimentally accessible core degradation stages (Figure S16).

Finally, in order to provide a qualitative comparison between the form factors extracted during this process and the experimental ones, we need to further consider the experimentally observed size increase as a function of $X$ and, accordingly, rescale each numerical form factor to obtain a comparison in $\mathrm{nm}^{-1}$.

Bulk Behavior. Microgels in bulk are investigated by means of molecular dynamics simulations with implicit solvent. Thermoresponsivity is mimicked by adding a solvophobic potential

$$
V_{\alpha}(r)= \begin{cases}-\epsilon \alpha & \text { if } r \leq 2^{1 / 6} \sigma \\ \frac{1}{2} \alpha \epsilon\left\{\cos \left[\delta\left(\frac{r}{\sigma}\right)^{2}+\beta\right]-1\right\} & \text { if } 2^{1 / 6} \sigma<r \leq R_{0} \sigma \\ 0 & \text { if } r>R_{0} \sigma\end{cases}
$$

with $\delta=\pi\left(\frac{9}{4}-2^{1 / 3}\right)^{-1}$ and $\beta=2 \pi-\frac{9}{4} \delta .{ }^{54} V_{\alpha}$ effectively introduces an attraction among all monomers of the network, modulated by the parameter $\alpha$ : in the case where $\alpha=0$, the standard KremerGrest model is recovered, whereas higher values of $\alpha$ mimic an increase in the temperature of the dispersion, leading to a microgel in the collapsed state at $\alpha \approx 1$.1. Simulations in bulk are performed in the NVT ensemble, fixing the reduced temperature $T^{*}=k_{\mathrm{B}} T / \epsilon=1$ with a Nosé-Hoover thermostat.

Interfacial Behavior. Investigations at the liquid-liquid interface are carried out in the presence of explicit solvent particles in order to reproduce the effect of the surface tension between the two solvents. Solvent particles are modeled as soft beads within the dissipative particle dynamics (DPD) framework. ${ }^{556}$ The total interaction force among beads is $\vec{F}_{i j}=\vec{F}_{i j}^{C}+\vec{F}_{i j}^{D}+\vec{F}_{i j}^{R}$, where

$$
\begin{aligned}
& \vec{F}_{i j}^{C}=a_{i j} w\left(r_{i j}\right) \hat{r}_{i j} \\
& \vec{F}_{i j}^{D}=-\gamma w^{2}\left(r_{i j}\right)\left(\vec{v}_{i j} \cdot \vec{r}_{i j}\right) \hat{r}_{i j} \\
& \vec{F}_{i j}^{R}=2 \gamma \frac{k_{\mathrm{B}} T}{m} w\left(r_{i j}\right) \frac{\theta}{\sqrt{\Delta t}} \hat{r}_{i j}
\end{aligned}
$$

where $\vec{F}_{i j}^{C}$ is a conservative repulsive force, with $w\left(r_{i j}\right)=1-r_{i j} / r_{c}$ for $r_{i j}$ $<r_{c}$ and 0 elsewhere, $\vec{F}_{i j}^{D}$ and $\vec{F}_{i j}^{R}$ are a dissipative and a random contribution of the DPD, respectively; $a_{i j}$ quantifies the repulsion between two particles, $\gamma=2.0$ is a friction coefficient, $\theta$ is a Gaussian random variable with zero average and unit variance, and $\Delta t=0.002$ is the integration time step. According to previous works, ${ }^{18,24}$ in order to reproduce a hexane-water interface, we choose $a_{\mathrm{ww}}=a_{\mathrm{hh}}=8.8, a_{\mathrm{hw}}$ $=31.1$. Instead, for the monomer-solvent interactions, we choose $a_{\mathrm{mw}}$ $=4.5$ and $a_{\mathrm{mh}}=5.0$. The cutoff radius is always set to be $r_{\mathrm{c}}=1.9 \sigma$ and the reduced solvent density $\rho_{\mathrm{DPD}}=4.5$. In this case, the reduced temperature $T^{*}$ is fixed to 1 via the DPD thermostat. At the interface, we limit the study to microgels with $Z=75 \sigma$ because of the exceptional computational cost to carry out the simulations in the presence of explicit solvent. Under these conditions, more than $5 \times$ $10^{6}$ solvent particles are inserted in the simulation box. Simulations are performed with the LAMMPS simulation package. ${ }^{57}$

For microgels adsorbed at the interface, we perform compression tests by imposing an external force of cylindrical symmetry, with the main axis perpendicular to the plane of the interface, along the $z$-axis. In this way, microgel monomers experience a harmonic force $F(r)=$ $-k(r-R)^{2}$ with $F(r)=0$ if $r>R$, where $r$ is the distance from the monomer to the center axis of the cylinder, $R$ is the equilibrium radius of the cylinder, and $k=10$ is the intensity of the force. Solvent particles are not subjected to $F(r)$.

Measured Quantities. In order to compare simulations and experiments, we calculate the numerical form factors in bulk as

$$
P(q)=\frac{1}{N} \sum_{i, j=1}^{N}\left\langle\exp \left(-i \vec{q} \cdot \vec{r}_{i j}\right)\right\rangle
$$

where $r_{i j}$ is the distance between monomers $i$ and $j$, while the angular brackets indicate an average over different configurations and over different orientations of the wavevector $\vec{q}$. From the rescaling factor between simulations and experiments, we get an effective size of the monomer bead, for which $\sigma=8.25 \mathrm{~nm}$. Although larger microgel sizes would be required to achieve a bead size comparable to the Kuhn length, ${ }^{21}$ to date, they cannot be easily handled in simulations. In real space, we calculate the radial density profiles of the microgel as

$$
\rho(r)=\left\langle\frac{1}{N} \sum_{i=1}^{N} \delta\left(\left|\vec{r}_{i}-\vec{r}_{C M}\right|-r\right)\right\rangle
$$

with $\vec{r}_{\mathrm{CM}}$ being the distance from the microgel center of mass. At the interface, similarly to that in ref 24 , we also determine $\rho(z)$, which is the density profile obtained by dividing the simulation box along the $z$-axis (with $z$ perpendicular to the interfacial plane) into threedimensional bins that are parallel to the interface, and $\rho(\zeta)$, with $\zeta=$ $x, y$, for which bins are taken orthogonally to the interfacial plane. The latter is calculated at a distance $\zeta$ from the microgel center of mass and averaged over $x$ and $y$. 


\section{ASSOCIATED CONTENT}

\section{(s) Supporting Information}

The Supporting Information is available free of charge at https://pubs.acs.org/doi/10.1021/acsnano.1c02486.

Additional data at different temperatures obtained by DLS and SLS, including fittings of the form factors; additional AFM images of individual microgels and microgel assemblies; calculated dry mass of adsorbed microgels as a function of core removal; additional AFM nanointendation results; top-view simulation snapshot of a hollow microgel adsorbed at a oil-water interface; plot of the center-to-center distance as a function of the area per particle in interfacial assemblies (PDF)

\section{AUTHOR INFORMATION}

\section{Corresponding Authors}

Lucio Isa - Laboratory for Soft Materials and Interfaces, Department of Materials, ETH Zürich, 8093 Zürich, Switzerland; (i) orcid.org/0000-0001-6731-9620;

Email: lucio.isa@mat.ethz.ch

Emanuela Zaccarelli - CNR Institute for Complex Systems, Uos Sapienza, 00185 Roma, Italy; Department of Physics, Sapienza University of Rome, 00185 Roma, Italy;

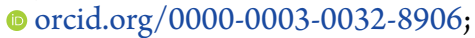

Email: emanuela.zaccarelli@cnr.it

Jacopo Vialetto - Laboratory for Soft Materials and Interfaces, Department of Materials, ETH Zürich, 8093 Zürich, Switzerland; 이이.org/0000-0002-4617-4386; Email: jacopo.vialetto@mat.ethz.ch

Fabrizio Camerin - CNR Institute for Complex Systems, Uos Sapienza, 00185 Roma, Italy; Department of Basic and Applied Sciences for Engineering, Sapienza University of Rome, 00161 Roma, Italy; Email: fabrizio.camerin@ gmail.com

\section{Authors}

Fabio Grillo - Laboratory for Soft Materials and Interfaces, Department of Materials, ETH Zürich, 8093 Zürich, Switzerland

Shivaprakash N. Ramakrishna - Laboratory for Soft Materials and Interfaces, Department of Materials, ETH Zürich, 8093 Zürich, Switzerland

Lorenzo Rovigatti - CNR Institute for Complex Systems, Uos Sapienza, 00185 Roma, Italy; Department of Physics, Sapienza University of Rome, 00185 Roma, Italy

Complete contact information is available at:

https://pubs.acs.org/10.1021/acsnano.1c02486

\section{Author Contributions}

Author contributions are defined based on the CRediT (Contributor Roles Taxonomy) and listed alphabetically. Conceptualization: L.I. and E.Z. Formal analysis: F.C., F.G., and J.V. Funding acquisition: L.I., J.V., and E.Z. Investigation: F.C., F.G., L.I., S.N.R., J.V., and E.Z. Methodology: F.C., F.G., L.R., J.V., and E.Z. Project administration: L.I. and E.Z. Software: F.C., F.G., and L.R. Supervision: L.I and E.Z. Validation: F.C. and J.V. Visualization: F.C., F.G., L.I., J.V., and E.Z. Writing-original draft: F.C., F.G., L.I., J.V., and E.Z. Writing-review and editing: F.C., F.G., L.I., L.R. J.V., and E.Z.

\section{Notes}

The authors declare no competing financial interest.

\section{ACKNOWLEDGMENTS}

We thank Miguel Angel Fernandez-Rodriguez for discussions. J.V. and L.I. acknowledge Dr. Kirill Feldman and Prof. Jan Vermant for instrumentation access and discussions. J.V. acknowledges funding from the European Union's Horizon 2020 research and innovation programme under the Marie Skłodowska Curie Grant Agreement 888076. F.C., L.R., and E.Z. acknowledge financial support from the European Research Council (ERC Consolidator Grant 681597, MIMIC) and gratefully acknowledge the computing time granted by EUSMI on the supercomputer JURECA at the Jülich Supercomputing Centre (JSC).

\section{REFERENCES}

(1) Velev, O. D.; Gupta, S. Materials Fabricated by Micro- and Nanoparticle Assembly - The Challenging Path from Science to Engineering. Adv. Mater. 2009, 21, 1897-1905.

(2) Vogel, N.; Retsch, M.; Fustin, C.-A.; del Campo, A.; Jonas, U. Advances in Colloidal Assembly: The Design of Structure and Hierarchy in Two and Three Dimensions. Chem. Rev. 2015, 115, 6265-6311

(3) Yunker, P. J.; Chen, K.; Gratale, M. D.; Lohr, M. A.; Still, T.; Yodh, A. G. Physics in Ordered and Disordered Colloidal Matter Composed of Poly(N-Isopropylacrylamide) Microgel Particles. Rep. Prog. Phys. 2014, 77, 056601.

(4) Rey, M.; Fernandez-Rodriguez, M. A.; Karg, M.; Isa, L.; Vogel, N. Poly-N-Isopropylacrylamide Nanogels and Microgels at Fluid Interfaces. Acc. Chem. Res. 2020, 53, 414-424.

(5) Pich, A.; Richtering, W. Microgels by Precipitation Polymerization: Synthesis, Characterization, and Functionalization. Chemical Design of Responsive Microgels. Advances in Polymer Science; Springer: Berlin, 2010; Vol. 234; pp 1-37.

(6) Plamper, F. A.; Richtering, W. Functional Microgels and Microgel Systems. Acc. Chem. Res. 2017, 50, 131-140.

(7) Karg, M.; Pich, A.; Hellweg, T.; Hoare, T.; Lyon, L. A.; Crassous, J. J.; Suzuki, D.; Gumerov, R. A.; Schneider, S.; Potemkin, I. I.; Richtering, W. Nanogels and Microgels: From Model Colloids to Applications, Recent Developments, and Future Trends. Langmuir 2019, 35, 6231-6255.

(8) Xia, Y.; He, X.; Cao, M.; Chen, C.; Xu, H.; Pan, F.; Lu, J. R. Thermoresponsive Microgel Films for Harvesting Cells and Cell Sheets. Biomacromolecules 2013, 14, 3615-3625.

(9) Kim, J.; Nayak, S.; Lyon, L. A. Bioresponsive Hydrogel Microlenses. J. Am. Chem. Soc. 2005, 127, 9588-9592.

(10) Tsuji, S.; Kawaguchi, H. Colored Thin Films Prepared from Hydrogel Microspheres. Langmuir 2005, 21, 8439-8442.

(11) Peng, J.; Zhao, D.; Tang, X.; Tong, F.; Guan, L.; Wang, Y.; Zhang, M.; Cao, T. Cool Microcontact Printing to Fabricate Thermosensitive Microgel Patterns. Langmuir 2013, 29, 1180911814.

(12) Rey, B. M.; Elnathan, R.; Ditcovski, R.; Geisel, K.; Zanini, M.; Fernandez-Rodriguez, M.-A.; Naik, V. V.; Frutiger, A.; Richtering, W.; Ellenbogen, T.; Voelcker, N. H.; Isa, L. Fully Tunable Silicon Nanowire Arrays Fabricated by Soft Nanoparticle Templating. Nano Lett. 2016, 16, 157-163.

(13) Fernández-Rodríguez, M. Á.; Elnathan, R.; Ditcovski, R.; Grillo, F.; Conley, G. M.; Timpu, F.; Rauh, A.; Geisel, K.; Ellenbogen, T.; Grange, R.; Scheffold, F.; Karg, M.; Richtering, W.; Voelcker, N. H.; Isa, L. Tunable 2D Binary Colloidal Alloys for Soft Nanotemplating. Nanoscale 2018, 10, 22189-22195.

(14) Fujii, S.; Mochizuki, M.; Aono, K.; Hamasaki, S.; Murakami, R.; Nakamura, Y. pH-Responsive Aqueous Foams Stabilized by Hairy Latex Particles. Langmuir 2011, 27, 12902-12909.

(15) Richtering, W. Responsive Emulsions Stabilized by StimuliSensitive Microgels: Emulsions with Special Non-Pickering Properties. Langmuir 2012, 28, 17218-17229. 
(16) Bergman, M. J.; Gnan, N.; Obiols-Rabasa, M.; Meijer, J.-M.; Rovigatti, L.; Zaccarelli, E.; Schurtenberger, P. A New Look at Effective Interactions between Microgel Particles. Nat. Commun. 2018, 9, 5039.

(17) Grillo, F.; Fernandez-Rodriguez, M. A.; Antonopoulou, M.-N.; Gerber, D.; Isa, L. Self-Templating Assembly of Soft Microparticles into Complex Tessellations. Nature 2020, 582, 219-224.

(18) Camerin, F.; Gnan, N.; Ruiz-Franco, J.; Ninarello, A.; Rovigatti, L.; Zaccarelli, E. Microgels at Interfaces Behave as 2D Elastic Particles Featuring Reentrant Dynamics. Phys. Rev. X 2020, 10, 031012

(19) Ciarella, S.; Rey, M.; Harrer, J.; Holstein, N.; Ickler, M.; Löwen, H.; Vogel, N.; Janssen, L. M. C. Soft Particles at Liquid Interfaces: From Molecular Particle Architecture to Collective Phase Behavior. Langmuir 2021, 37, 5364-5375.

(20) Bergmann, S.; Wrede, O.; Huser, T.; Hellweg, T. SuperResolution Optical Microscopy Resolves Network Morphology of Smart Colloidal Microgels. Phys. Chem. Chem. Phys. 2018, 20, 50745083

(21) Ninarello, A.; Crassous, J. J.; Paloli, D.; Camerin, F.; Gnan, N.; Rovigatti, L.; Schurtenberger, P.; Zaccarelli, E. Modeling Microgels with a Controlled Structure across the Volume Phase Transition. Macromolecules 2019, 52, 7584-7592.

(22) Geisel, K.; Isa, L.; Richtering, W. Unraveling the 3D Localization and Deformation of Responsive Microgels at Oil/ Water Interfaces: A Step Forward in Understanding Soft Emulsion Stabilizers. Langmuir 2012, 28, 15770-15776.

(23) Geisel, K.; Rudov, A. A.; Potemkin, I. I.; Richtering, W. Hollow and Core-Shell Microgels at Oil-Water Interfaces: Spreading of Soft Particles Reduces the Compressibility of the Monolayer. Langmuir 2015, 31, 13145-13154.

(24) Camerin, F.; Fernandez-Rodríguez, M. A.; Rovigatti, L.; Antonopoulou, M.-N.; Gnan, N.; Ninarello, A.; Isa, L.; Zaccarelli, E. Microgels Adsorbed at Liquid-Liquid Interfaces: A Joint Numerical and Experimental Study. ACS Nano 2019, 13, 4548-4559.

(25) Acciaro, R.; Gilányi, T.; Varga, I. Preparation of Monodisperse Poly(N-Isopropylacrylamide) Microgel Particles with Homogenous Cross-Link Density Distribution. Langmuir 2011, 27, 7917-7925.

(26) Still, T.; Chen, K.; Alsayed, A. M.; Aptowicz, K. B.; Yodh, A. Synthesis of Micrometer-Size Poly(N-Isopropylacrylamide) Microgel Particles with Homogeneous Crosslinker Density and Diameter Control. J. Colloid Interface Sci. 2013, 405, 96-102.

(27) Zha, L.; Zhang, Y.; Yang, W.; Fu, S. Monodisperse Temperature-Sensitive Microcontainers. Adv. Mater. 2002, 14, 1090.

(28) Nayak, S.; Gan, D.; Serpe, M. J.; Lyon, L. A. Hollow Thermoresponsive Microgels. Small 2005, 1, 416-421.

(29) Dubbert, J.; Honold, T.; Pedersen, J. S.; Radulescu, A.; Drechsler, M.; Karg, M.; Richtering, W. How Hollow Are Thermoresponsive Hollow Nanogels? Macromolecules 2014, 47, $8700-8708$.

(30) Zhang, F.; Wang, C.-C. Preparation of Thermoresponsive Core-Shell Polymeric Microspheres and Hollow PNIPAM. Colloid Polym. Sci. 2008, 286, 889-895.

(31) Schulte, M. F.; Scotti, A.; Gelissen, A. P. H.; Richtering, W.; Mourran, A. Probing the Internal Heterogeneity of Responsive Microgels Adsorbed to an Interface by a Sharp SFM Tip: Comparing Core-Shell and Hollow Microgels. Langmuir 2018, 34, 4150-4158.

(32) Nickel, A. C.; Scotti, A.; Houston, J. E.; Ito, T.; Crassous, J.; Pedersen, J. S.; Richtering, W. Anisotropic Hollow Microgels That Can Adapt Their Size, Shape, and Softness. Nano Lett. 2019, 19, $8161-8170$

(33) Scotti, A.; Denton, A. R.; Brugnoni, M.; Houston, J. E.; Schweins, R.; Potemkin, I. I.; Richtering, W. Deswelling of Microgels in Crowded Suspensions Depends on Cross-Link Density and Architecture. Macromolecules 2019, 52, 3995-4007.

(34) Schmid, A. J.; Dubbert, J.; Rudov, A. A.; Pedersen, J. S.; Lindner, P.; Karg, M.; Potemkin, I. I.; Richtering, W. Multi-Shell Hollow Nanogels with Responsive Shell Permeability. Sci. Rep. 2016, 6, 22736.
(35) Scotti, A.; Brugnoni, M.; Rudov, A. A.; Houston, J. E.; Potemkin, I. I.; Richtering, W. Hollow Microgels Squeezed in Overcrowded Environments. J. Chem. Phys. 2018, 148, 174903.

(36) Geisel, K.; Richtering, W.; Isa, L. Highly Ordered 2D Microgel Arrays: Compression versus Self-Assembly. Soft Matter 2014, 10, 7968-7976.

(37) Schulte, M. F.; Bochenek, S.; Brugnoni, M.; Scotti, A.; Mourran, A.; Richtering, W. Stiffness Tomography of Ultra-Soft Nanogels by Atomic Force Microscopy. Angew. Chem., Int. Ed. 2021, $60,2280-2287$

(38) Scotti, A.; Bochenek, S.; Brugnoni, M.; Fernandez-Rodriguez, M. A.; Schulte, M. F.; Houston, J. E.; Gelissen, A. P. H.; Potemkin, I. I.; Isa, L.; Richtering, W. Exploring the Colloid-to-Polymer Transition for Ultra-Low Crosslinked Microgels from Three to Two Dimensions. Nat. Commun. 2019, 10, 1418.

(39) Picard, C.; Garrigue, P.; Tatry, M.-c.; Lapeyre, V.; Ravaine, S.; Schmitt, V.; Ravaine, V. Organization of Microgels at the Air-Water Interface under Compression: Role of Electrostatics and CrossLinking Density. Langmuir 2017, 33, 7968-7981.

(40) Rey, M.; Hou, X.; Tang, J. S. J.; Vogel, N. Interfacial Arrangement and Phase Transitions of PNiPAm Microgels With Different Crosslinking Densities. Soft Matter 2017, 13, 8717-8727.

(41) Scheidegger, L.; Fernández-Rodríguez, M. A.; Geisel, K.; Zanini, M.; Elnathan, R.; Richtering, W.; Isa, L. Compression and Deposition of Microgel Monolayers from Fluid Interfaces: Particle Size Effects on Interface Microstructure and Nanolithography. Phys. Chem. Chem. Phys. 2017, 19, 8671-8680.

(42) Clegg, P. S.; Herzig, E. M.; Schofield, A. B.; Egelhaaf, S. U.; Horozov, T. S.; Binks, B. P.; Cates, M. E.; Poon, W. C. Emulsification of Partially Miscible Liquids Using Colloidal Particles: Nonspherical and Extended Domain Structures. Langmuir 2007, 23, 5984-5994.

(43) Rey, M.; Fernández-Rodríguez, M. Á.; Steinacher, M.; Scheidegger, L.; Geisel, K.; Richtering, W.; Squires, T. M.; Isa, L. Isostructural Solid-Solid Phase Transition in Monolayers of Soft Core-Shell Particles at Fluid Interfaces: Structure and Mechanics. Soft Matter 2016, 12, 3545-3557.

(44) Scotti, A.; Denton, A.; Brugnoni, M.; Schweins, R.; Richtering, W. Absence of Crystals in the Phase Behavior of Hollow Microgels. Phys. Rev. E: Stat. Phys., Plasmas, Fluids, Relat. Interdiscip. Top. 2021, 103, 022612.

(45) Virtanen, O. L. J.; Mourran, A.; Pinard, P. T.; Richtering, W. Persulfate Initiated Ultra-Low Cross-Linked Poly(N-Isopropylacrylamide) Microgels Possess an Unusual Inverted Cross-Linking Structure. Soft Matter 2016, 12, 3919-3928.

(46) Isa, L.; Kumar, K.; Müller, M.; Grolig, J.; Textor, M.; Reimhult, E. Particle Lithography from Colloidal Self-Assembly at Liquid-Liquid Interfaces. ACS Nano 2010, 4, 5665-5670.

(47) Harper, E. S.; Spellings, M.; Anderson, J.; Glotzer, S. C. Zenodo, version v0.6.1, 2016.

(48) Miller, W. L.; Cacciuto, A. Two-Dimensional Packing of Soft Particles and the Soft Generalized Thomson Problem. Soft Matter 2011, 7, 7552-7559.

(49) Zu, M.; Tan, P.; Xu, N. Forming Quasicrystals by Monodisperse Soft Core Particles. Nat. Commun. 2017, 8, 2089.

(50) Hutter, J. L.; Bechhoefer, J. Calibration of Atomic-Force Microscope Tips. Rev. Sci. Instrum. 1993, 64, 1868-1873.

(51) Gnan, N.; Rovigatti, L.; Bergman, M.; Zaccarelli, E. In Silico Synthesis of Microgel Particles. Macromolecules 2017, 50, 8777-8786.

(52) Rovigatti, L.; Sulc, P.; Reguly, I. Z.; Romano, F. A Comparison between Parallelization Approaches in Molecular Dynamics Simulations on GPUs. J. Comput. Chem. 2015, 36, 1-8.

(53) Kremer, K.; Grest, G. S. Dynamics of Entangled Linear Polymer Melts: A Molecular-Dynamics Simulation. J. Chem. Phys. 1990, 92, 5057-5086.

(54) Soddemann, T.; Dünweg, B.; Kremer, K. A Generic Computer Model for Amphiphilic Systems. Eur. Phys. J. E: Soft Matter Biol. Phys. 2001, 6, 409-419. 
(55) Groot, R. D.; Warren, P. B. Dissipative Particle Dynamics: Bridging the Gap between Atomistic and Mesoscopic Simulation. J. Chem. Phys. 1997, 107, 4423-4435.

(56) Camerin, F.; Gnan, N.; Rovigatti, L.; Zaccarelli, E. Modelling Realistic Microgels in an Explicit Solvent. Sci. Rep. 2018, 8, 14426.

(57) Plimpton, S. Fast Parallel Algorithms for Short-Range Molecular Dynamics. J. Comput. Phys. 1995, 117, 1-19. 